\title{
Hepatocellular Carcinoma Risk According to Regimens for Eradication of Hepatitis C Virus; Interferon or Direct Acting Antivirals
}

\author{
Hye Won Lee ${ }^{1,2,3,4,+} \mathbb{D}$, Dai Hoon Han ${ }^{3,4,5,+} \mathbb{D}$, Hye Jung Shin ${ }^{6} \mathbb{D}$, Jae Seung Lee ${ }^{1,2,3,4} \mathbb{D}^{\mathbb{D}}$, \\ Seung Up Kim ${ }^{1,2,3,4}\left(\mathbb{D}\right.$, Jun Yong Park ${ }^{1,2,3,4} \mathbb{D}^{\text {, Do Young Kim }}{ }^{1,2,3,4}$, Sang Hoon Ahn $1,2,3,4$ \\ and Beom Kyung Kim 1,2,3,4,* \\ 1 Department of Internal Medicine, Yonsei University College of Medicine, Seoul 03722, Korea; \\ lorry-lee@yuhs.ac (H.W.L.); sikarue@yuhs.ac (J.S.L.); ksukorea@yuhs.ac (S.U.K.); drpjy@yuhs.ac (J.Y.P.); \\ dyk1025@yuhs.ac (D.Y.K.); ahnsh@yuhs.ac (S.H.A.) \\ 2 Institute of Gastroenterology, Yonsei University College of Medicine, Seoul 03722, Korea \\ 3 Yonsei Liver Center, Severance Hospital, Seoul 03722, Korea; dhhan@yuhs.ac \\ 4 Liver Cancer Center, Yonsei Cancer Center, Yonsei University Health System, Seoul 03722, Korea \\ 5 Division of Hepatobiliary and Pancreatic Surgery, Department of Surgery, Yonsei University College of \\ medicine, Seoul 03722, Korea \\ 6 Biostatistics Collaboration Unit, Department of Biomedical Systems Informatics, Yonsei University College \\ of Medicine, Seoul 03722, Korea; hjshin105@yuhs.ac \\ * Correspondence: beomkkim@yuhs.ac; Tel.: +82-2-2228-1930; Fax: +82-2-393-6884 \\ + Both two authors equally contributed to the work as co-first authors.
}

Received: 20 October 2020; Accepted: 16 November 2020; Published: 18 November 2020

Simple Summary: Owing to pegylated interferon (PegIFN)-free direct-acting antivirals (DAAs) against chronic hepatitis $C$ virus infection, a sustained virological response (SVR) rate $>95 \%$ can be attained with a satisfactory tolerability and shorter treatment duration. However, it still remains controversial whether there is any difference in prognosis depending on treatment regimens-PegIFN or DAAs. After adjusting for imbalance between patients treated with PegIFN-based vs. DAA-based regimens, the post-SVR risk of hepatocellular carcinoma development was comparable according to treatment regimens. Furthermore, the risk was also similar between patients treated with sofosbuvir-based vs. sofosbuvir-free DAA regimens. Further studies with a longer follow-up period are required.

\begin{abstract}
By pegylated interferon (PegIFN)-free direct-acting antivirals (DAAs) against hepatitis $\mathrm{C}$ virus (HCV) infection, a sustained virological response (SVR) rate $>95 \%$ can be attained with a satisfactory tolerability and shorter treatment duration. However, it remains controversial whether there is any difference in prognosis depending on regimens-PegIFN or DAAs. We compared the probabilities of hepatocellular carcinoma (HCC) development between patients achieving an SVR by PegIFN/ribavirin (PegIFN group, $n=603$ ) and DAAs (DAAs group, $n=479$ ). The DAAs group was significantly older and had a higher proportion of cirrhosis than the PegIFN group. Before adjustment, the DAAs group had a higher HCC incidence than the PegIFN group $(p<0.001)$. However, by multivariate analyses, the DAAs (vs. PegIFN) group was not associated with HCC risk (adjusted hazard ratio $0.968,95 \%$ confidence interval 0.380-2.468; $p=0.946$ ). Old age, male, higher body mass index, cirrhosis, and lower platelet count were associated with increased HCC risk (all $p<0.05$ ). After propensity score matching (PSM), a similar HCC risk between the two groups was observed ( $p=0.372$ ). We also compared HCC incidences according to sofosbuvir (SOF)-based and SOF-free DAAs, showing a similar risk in both groups before adjustment $(p=0.478)$ and after PSM $(p=0.855)$. In conclusion, post-SVR HCC risks were comparable according to treatment regimens; PegIFN- vs. DAA-based regimens and SOF-based vs. SOF-free DAA regimens. Further studies with a longer follow-up period are required.
\end{abstract}


Keywords: interferon; direct-acting antivirals; hepatocellular carcinoma; prognosis; comparison

\section{Introduction}

Hepatitis $\mathrm{C}$ virus (HCV) is globally one of the major health problems causing hepatocellular carcinoma (HCC) and/or cirrhosis [1]. Successful eradication of chronic HCV infection through antiviral treatment has proved to reduce the risk of such liver disease progression. Previously, antiviral treatments centered on the use of interferon-alpha (IFN)-based regimens. However, pegylated IFN (PegIFN)/ribavirin (RBV) not only has various kinds of adverse effects with a long dose administration period, i.e., at least 24 to 48 weeks, but also an inevitably high rate of treatment discontinuation. Therefore, its wide use is substantially limited, especially for patients with old age, decompensated cirrhosis, or other comorbidities. In addition, more importantly, their sustained virological response (SVR) rates are also sub-optimal. Currently, primarily owing to the introduction of PegIFN-free direct-acting antivirals (DAAs) in 2014, an SVR rate $>95 \%$ can be attained with satisfactory tolerability [2-7].

Meanwhile, the risk of HCC development has not been well-controlled in real-life practice, even after achievement of SVR. In the early period since PegIFN-free DAA regimens became available, data showed the paradoxically tumorigenic potential, facilitating the early occurrence and recurrence of HCC following a DAA-induced SVR [8,9]. Furthermore, in an article about cost-effective analysis of post-SVR HCC surveillance, HCC incidences after DAA-induced SVR were adopted at $0.34 \sim 1.82 \%$ /years, slightly higher than those after IFN-induced SVR of 0.23 1.39\%/years [10]. Several molecular studies theoretically supported such a phenomenon by indicating the potential effect of DAAs on angiogenesis and cellular proliferation [11-13]. In a similar context, another study reported that sofosbuvir (SOF) may activate epidermal growth factor receptor (EGFR)-dependent pathways, inducing liver-related pathological processes [14]. Nevertheless, subsequent studies have failed to reproduce such kinds of data systematically yet, indicating that there has been no difference in the risk of HCC occurrence or recurrence following SVR from DAA- vs. IFN-based therapy so far [15-18]. However, Ogawa et al. [19] recently proposed a trend toward a higher short-term HCC incidence in a subgroup treated with a specific DAA, i.e., SOF.

In this study, we aimed to compare the preventive effect of HCV eradication by PegIFN/RBV vs. DAAs on liver disease progression, including the risk of HCC development, and to assess differences in HCC incidence according to the type of DAA.

\section{Methods}

\subsection{Patients}

From 2011 to 2019, treatment-naïve patients with chronic HCV infection who were treated with PegIFN /RBV regimen or DAAs (referred as "PegIFN group" and "DAAs group", respectively) at the Severance Hospital, Yonsei University College of Medicine, Seoul, Republic of Korea, were screened for eligibility. The inclusion criteria were as follows: (1) age $\geq 19$ years; (2) detectable serum HCV-RNA before antiviral treatment; and (3) achievement of SVR after antiviral treatment. Exclusion criteria were as follows: (1) simultaneous administration of PegIFN plus DAAs; (2) previous antiviral treatment against HCV infection; (3) history of HCC or hepatic decompensation; (4) co-infection with other hepatitis viruses; (5) reinfection after SVR; (6) history of organ transplantation; (7) HCC development, hepatic decompensation, or death within 6 months of enrollment; and (8) other significant medical illnesses. PegIFN/RBV or DAA regimens were prescribed according to the practice guideline $[20,21]$ and SVR was defined as undetectable serum HCV-RNA with a lower limit of quantification of $15 \mathrm{IU} / \mathrm{mL}$ at 24 or 12 weeks after PegIFN/RBV or DAA regimens, respectively. If histologic information was not available, cirrhosis was clinically defined as follows: (1) platelet count $<150,000 / \mu \mathrm{L}$ and 
ultrasonographic findings suggestive of cirrhosis, including a blunted, nodular liver edge accompanied by splenomegaly ( $>12 \mathrm{~cm})$; or (2) esophageal or gastric varices [22].

The study was approved by the institutional review board of the Severance Hospital and conformed to the ethical guidelines of the 1975 Helsinki Declaration (IRB No. 4-2017-1217).

\subsection{Clinical Evaluation and Study Endpoints}

After achievement of SVR, all patients received laboratory tests every 6 months and underwent periodic surveillance with ultrasonography and serum alpha-fetoprotein levels to screen for HCC and cirrhotic complications every 6 months [23-25].

The primary outcome was the cumulative risk of HCC development. HCC was diagnosed based on histological evidence or radiological findings determined by dynamic computed tomography and/or magnetic resonance imaging (nodule $>1 \mathrm{~cm}$ with arterial hyper-vascularity and portal/delayed-phase washout) [26-29]. In addition, we also investigated the cumulative risk of cirrhotic complication events (CCEs), including shift to Child-Pugh class B, presence of related symptoms (e.g., ascites, variceal hemorrhage, hepatorenal syndrome, or hepatic encephalopathy), cirrhosis-related death, or liver transplantation. To avoid statistical repetition in a patient experiencing different types of cirrhotic complications at different times, we selected the first event of cirrhotic complications for statistical analysis.

\subsection{Statistical Analysis}

Data are expressed as means \pm standard deviations, medians (IQRs), or no. (\%), as appropriate. Differences among continuous and categorical variables were examined for statistical significance by the Student's $t$-test (or the Mann-Whitney test, if appropriate) and chi-squared (or Fisher's exact tests, if appropriate) test.

The cumulative risk of development of HCC or CCEs was evaluated using the Kaplan-Meier method, with comparisons using the log-rank test. As DAAs in the Republic of Korea became widely prescribed since 2015, the follow-up was censored at 48 months. In order to assess the associations between the risk of HCC or CCEs and each variable and to calculate hazard ratios (HRs) and 95\% confidence intervals (CIs), a Cox regression analysis was performed. Then, a multivariable Cox regression analysis was performed to determine the final prognostic factors associated with disease progression. Furthermore, to reduce the effect of selection bias and potential confounders between the PegIFN and DAA groups, propensity score (PS) was calculated using logistic regression. Then, PS matching analyses were performed to compare the cumulative risks of HCC or CCEs between the two groups.

All statistical analyses were conducted using SAS, version 9.2 (SAS Institute), R (V.3.0, http://cran.r-project.org/), and IBM ${ }^{\circledR}$ SPSS ${ }^{\circledR}$ Statistics for Windows, version 25.0 (IBM Corp., Armonk, NY, USA). Two-sided $p$-values $<0.05$ were considered to indicate statistical significance.

\section{Results}

\subsection{Patients' Characteristics}

According to the recruitment criteria, a total of 1082 patients were analyzed (Figure S1). Patients' characteristics at the time of SVR are described in Table 1. The DAAs group $(n=603)$ members were significantly older (mean 61.0 vs. 49.5 years, respectively; $p<0.001$ ) and had a lower proportion of males (38.0 vs. $44.7 \%$, respectively; $p=0.029$ ), lower aspartate aminotransferase (AST) (mean 25.0 vs. $32.5 \mathrm{IU} / \mathrm{mL}$, respectively; $p<0.001$ ), alanine aminotransferase (ALT) (mean $19.7 \mathrm{vs.} 29.1 \mathrm{IU} / \mathrm{mL}$, respectively; $p<0.001$ ), and albumin (mean $4.1 \mathrm{vs.} 4.3 \mathrm{~g} / \mathrm{dL}$, respectively; $p<0.001$ ) levels, and higher total bilirubin (mean 0.9 vs. $0.7 \mathrm{mg} / \mathrm{dL}$, respectively; $p<0.001$ ), platelet count levels (mean 186 vs. $161 \times 10^{3} / \mathrm{uL}$, respectively; $p<0.001$ ), and alpha-fetoprotein (AFP) (median $3.26 \mathrm{vs.} 2.45 \mathrm{ng} / \mathrm{mL}$, respectively), in comparison with the PegIFN group $(n=479)$. The proportion of HCV genotype 2 was 
significantly higher in the PegIFN group than in the DAAs group (60.8 vs. $46.3 \%$, respectively; $p<0.001$ ). The most commonly used first-line DAAs regimen was daclatasvir/asunaprevir (36.8\%), followed by SOF/RBV (34.2\%) (Table 2). Among the entire population, the most common HCV genotype was 2 $(n=570)$, followed by genotyped $1(n=496), 3(n=14), 4(n=1)$, and $6(n=1)$.

Table 1. Comparison of clinical characteristics at the time of sustained virological response (SVR) between two groups $(n=1082)$.

\begin{tabular}{|c|c|c|c|c|}
\hline Variables & Total $(n=1082)$ & $\begin{array}{l}\text { DAAs Group } \\
\quad(n=603)\end{array}$ & $\begin{array}{l}\text { PegIFN Group } \\
\quad(n=479)\end{array}$ & $p$ Value \\
\hline Age, years & $55.9 \pm 13.7$ & $61.0 \pm 12.8$ & $49.5 \pm 12.0$ & $<0.001$ \\
\hline Male gender, no. (\%) & $443(40.9)$ & $229(38.0)$ & $214(44.7)$ & 0.029 \\
\hline Body mass index, $\mathrm{kg} / \mathrm{m}^{2}$ & $23.8 \pm 3.3$ & $23.8 \pm 3.4$ & $23.7 \pm 3.2$ & 0.831 \\
\hline Diabetes, no. $(\%)$ & $240(22.2)$ & $145(24.0)$ & $95(19.8)$ & 0.056 \\
\hline AST, IU/mL & $28.3 \pm 21.8$ & $25.0 \pm 13.5$ & $32.5 \pm 28.5$ & $<0.001$ \\
\hline ALT, IU/mL & $23.9 \pm 22.3$ & $19.7 \pm 14.9$ & $29.1 \pm 28.1$ & $<0.001$ \\
\hline Total bilirubin, mg/dL & $0.8 \pm 0.4$ & $0.9 \pm 0.5$ & $0.7 \pm 0.3$ & $<0.001$ \\
\hline Albumin, g/dL & $4.2 \pm 0.4$ & $4.1 \pm 0.4$ & $4.3 \pm 0.4$ & $<0.001$ \\
\hline Platelet count, $\times 10^{3} / \mathrm{uL}$ & $175 \pm 70$ & $186 \pm 72$ & $161 \pm 64$ & $<0.001$ \\
\hline Creatinine, mg/dL & $0.9 \pm 1.1$ & $0.9 \pm 1.0$ & $0.9 \pm 1.1$ & 0.870 \\
\hline $\mathrm{AFP}, \mathrm{ng} / \mathrm{mL}$ & $2.91(2.05 \sim 4.35)$ & $3.26(2.35 \sim 4.86)$ & $2.45(1.82 \sim 3.55)$ & $<0.001$ \\
\hline FIB-4 index & $1.80(1.16 \sim 3.00)$ & $1.86(1.18 \sim 3.09)$ & $1.73(1.11 \sim 2.91)$ & 0.142 \\
\hline Liver cirrhosis, no. (\%) & $247(22.8)$ & $165(27.4)$ & $82(17.1)$ & $<0.001$ \\
\hline HCV genotype 2, no. (\%) & $570(52.7)$ & 279 (46.3) & $291(60.8)$ & $<0.001$ \\
\hline
\end{tabular}

Table 2. Types of DAAs $(n=603)$.

\begin{tabular}{cc}
\hline DAAs & No. (\%) \\
\hline Daclatasvir + asunaprevir & $222(36.8)$ \\
SOF + RBV & $206(34.2)$ \\
Glecaprevir + pibrentasvir & $49(8.1)$ \\
Elbasvir + grazoprevir & $44(7.3)$ \\
Ledipasvir + SOF & $41(6.8)$ \\
Ombitasvir + paritaprevir + ritonavir + dasabuvir & $41(6.8)$ \\
\hline
\end{tabular}

Abbreviations: DAAs, direct-acting antivirals; $\mathrm{SOF}$, sofosbuvir; RBV, ribavirin.

\subsection{Changes in the Fibrotic Burden before and after Treatments}

We assessed the fibrotic burden before and after treatment, using the FIB-4 index [30]. Before treatment, the DAAs group had a significantly higher FIB-4 index than the PegIFN group (2.62 [IQR $1.47 \sim 5.33$ ] vs. 1.67 [IQR 0.97 3.04], respectively; $p<0.001$ ). However, the DAAs group showed a significantly favorable difference rate of FIB-4 index from the pre-treatment value than the PegIFN group (23.9\% [IQR 5.3 44.7] vs. $-11.0 \%$ [IQR -52.6 23.9], respectively; $p<0.001$ ). Consequently, there was no significant difference in the post-SVR FIB-4 index between the DAAs and PegIFN groups-1.86 (IQR 1.18 3.09) vs. 1.73 (IQR 1.11 2.91), respectively $(p=0.142)$.

\subsection{Predictive Factors for HCC and CCE Development}

During the follow-up, a total of $33(3.0 \%)$ and $47(4.3 \%)$ patients developed HCC and CCE, respectively. Before statistical adjustment, the cumulative probabilities of both HCC (Figure 1A) and CCE (Figure 1B) development were significantly higher in the DAAs group than in the PegIFN group (both $p<0.001$ ). To adjust bias between two groups, multivariate analyses were performed on the basis of significant univariate predictors with $p<0.05$ (Table 3). In terms of the risk of HCC development, antiviral regimens (DAAs vs. PegIFN groups) did not affect the final prognosis, with adjusted HR 
(aHR) of 0.968 (95\% CI 0.380 2.468; $p=0.946)$. Instead, old age (aHR 1.104, 95\% CI 1.055 1.155; $p<0.001$ ), male (aHR 2.990, 95\% CI 1.365 6.550; $p=0.006)$, body mass index (aHR 1.127, 95\% CI 1.023 1.241, $p=0.015$ ), liver cirrhosis (aHR 4.861, 95\% CI 1.706 13.849; $p=0.003$ ) and lower platelet count (aHR 0.988, 95\% CI 0.977 0.998; $p=0.023$ ) at the time of SVR were independently associated with an increased risk of HCC development.

A

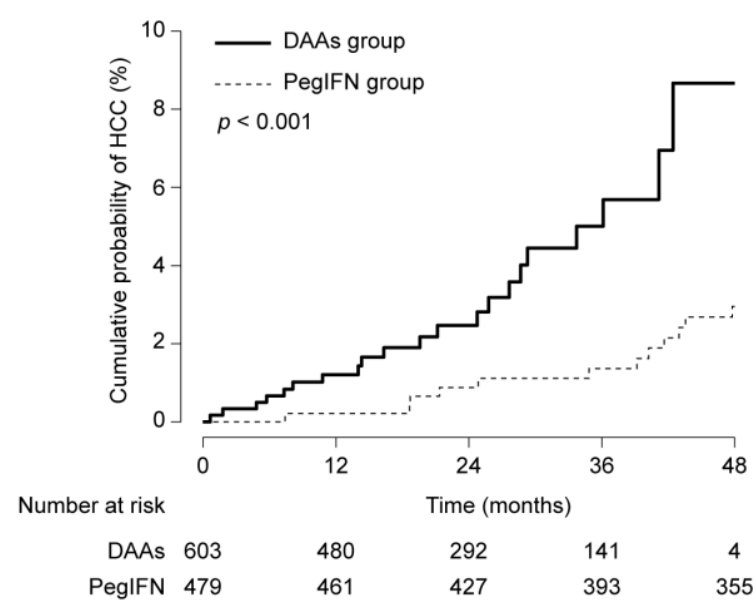

B

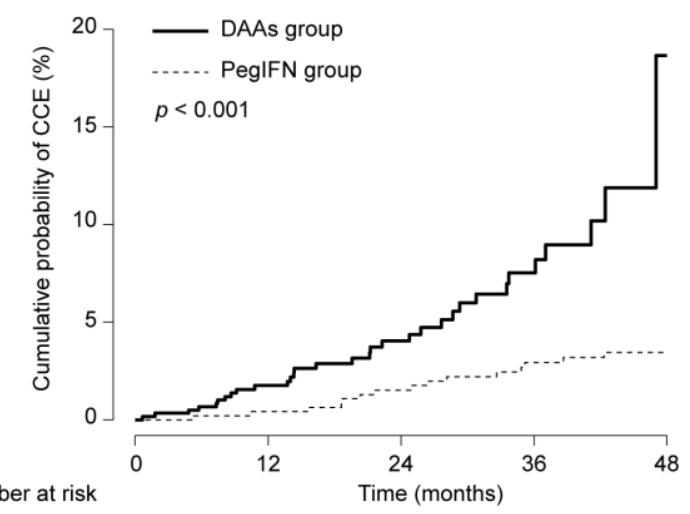

Number at risk

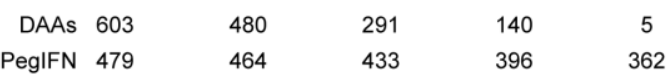

Figure 1. Cumulative probabilities of hepatocellular carcinoma (HCC) (A) and cirrhotic complication event (CCE) (B) development between the DAAs and PegIFN groups before statistical adjustment.

The contents of CCEs were as follows; varix bleeding $(n=11)$, ascites $(n=32)$, hepatic encephalopathy $(n=2)$, hepatorenal syndrome $(n=1)$, or liver transplantation $(n=1)$. Likewise, in terms of the risk of CCE development, antiviral regimens (DAAs vs. PegIFN groups) did not affect the final prognosis, with an aHR of 1.840 (95\% CI 0.858 3.945; $p=0.117)$. Instead, old age (aHR 1.035, 95\% CI 1.003 1.067; $p=0.032$ ), diabetes (aHR 1.815, 95\% CI 1.013 3.251; $p=0.045$ ), and liver cirrhosis (aHR 2.267, 95\% CI 1.095 4.696; $p=0.028$ ) at the time of SVR were independently associated with an increased risk of CCE development. 
Table 3. Predictive factors for clinical outcomes $(n=1082)$.

\begin{tabular}{|c|c|c|c|c|c|c|c|c|}
\hline \multirow[b]{3}{*}{ Variables } & \multicolumn{4}{|c|}{ HCC Development } & \multicolumn{4}{|c|}{ CCE Development } \\
\hline & \multirow{2}{*}{$\begin{array}{c}\text { Univariate } \\
p \text { Values }\end{array}$} & \multicolumn{3}{|c|}{ Multivariate } & \multirow{2}{*}{$\begin{array}{c}\text { Univariate } \\
p \text { Values }\end{array}$} & \multicolumn{3}{|c|}{ Multivariate } \\
\hline & & Adjusted HR & $95 \% \mathrm{CI}$ & $p$ Values & & Adjusted HR & $95 \% \mathrm{CI}$ & $p$ Values \\
\hline Age & $<0.001$ & 1.104 & $1.055-1.155$ & $<0.001$ & $<0.001$ & 1.035 & $1.003-1.067$ & 0.032 \\
\hline Male gender & 0.006 & 2.990 & $1.365-6.550$ & 0.006 & 0.063 & - & - & - \\
\hline BMI, $\mathrm{kg} / \mathrm{m}^{2}$ & 0.004 & 1.127 & $1.023-1.241$ & 0.015 & 0.127 & - & - & - \\
\hline Diabetes & $<0.001$ & 2.002 & $0.946-4.238$ & 0.070 & 0.001 & 1.815 & $1.013-3.251$ & 0.045 \\
\hline Liver cirrhosis & $<0.001$ & 4.861 & $1.706-13.849$ & 0.003 & $<0.001$ & 2.267 & $1.095-4.696$ & 0.028 \\
\hline FIB-4 index & $<0.001$ & 0.949 & 0.827-1.090 & 0.460 & $<0.001$ & 1.002 & $0.906-1.108$ & 0.971 \\
\hline Total bilirubin & 0.009 & 0.823 & $0.316-2.145$ & 0.690 & $<0.001$ & 1.298 & $0.672-2.505$ & 0.438 \\
\hline Albumin & $<0.001$ & 0.783 & $0.321-1.912$ & 0.592 & $<0.001$ & 0.603 & $0.291-1.249$ & 0.174 \\
\hline Platelet count & $<0.001$ & 0.988 & $0.977-0.998$ & 0.023 & $<0.001$ & 0.996 & $0.989-1.003$ & 0.259 \\
\hline $\mathrm{AFP}, \mathrm{ng} / \mathrm{mL}$ & 0.566 & - & - & - & 0.641 & - & - & - \\
\hline DAAs (vs. PegIFN) group & 0.001 & 0.968 & $0.380-2.468$ & 0.946 & $<0.001$ & 1.840 & $0.858-3.945$ & 0.117 \\
\hline
\end{tabular}

Abbreviations: HCC, hepatocellular carcinoma; CCE, cirrhotic complication event; HR, hazard ratio; 95\% CI, 95\% confidence interval; BMI, body mass index; FIB-4, fibrosis-4 AFP, alpha-fetoprotein; DAAs, direct-acting antivirals; PegIFN, pegylated interferon. 


\subsection{The Risk of HCC and CCE Development after PS Matching Analysis}

PS was calculated based upon variables at the time of SVR; age, male gender, diabetes, ALT, albumin, total bilirubin, platelet count, and liver cirrhosis. PS matching with a 1:1 ratio generated 182 pairs, where variables were well-balanced (all $p>0.05$ ). Like the results shown from multivariate analyses, similar phenomena were also observed; there was no significant difference in terms of the risk of HCC (Figure 2A; $p=0.372$ ) and CCE (Figure 2B; $p=0.723$ ) development.

A

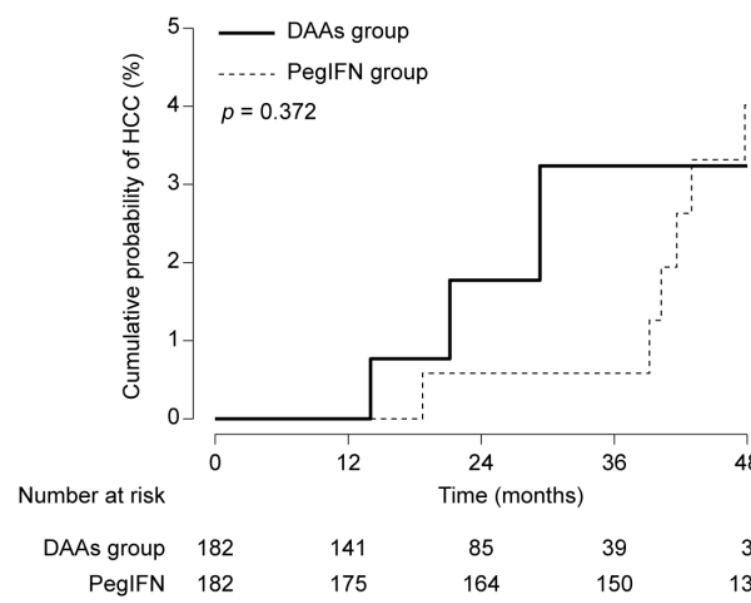

B

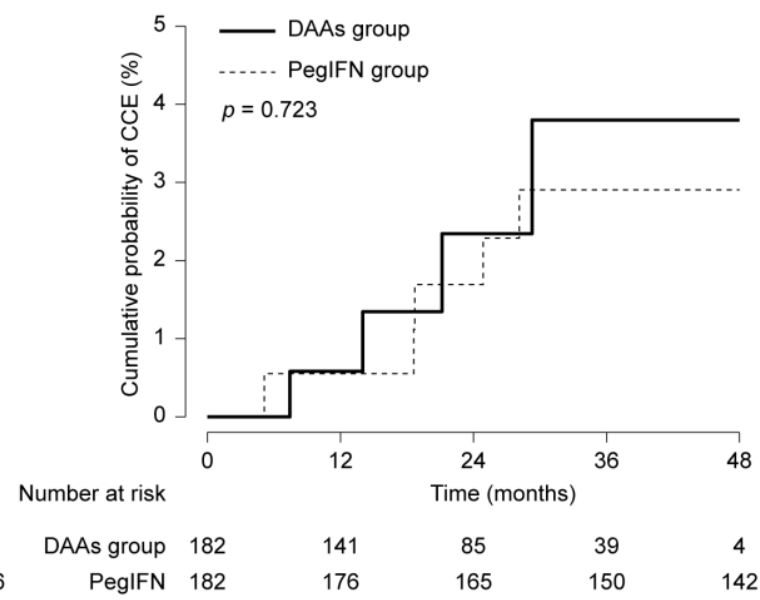

Figure 2. Cumulative probabilities of HCC (A) and CCE (B) development between the DAAs and PegIFN groups after propensity score (PS) matching analysis.

\subsection{Subgroup Analyses among the DAAs Group}

Among the DAAs group, we also compared the risk of HCC and CCE development according to the specific DAA regimen, i.e., SOF. The clinical characteristics of patients treated with the SOF-based DAAs regimen $(n=206)$ and with the SOF-free DAAs regimen $(n=397)$ are described in Table 4. Before adjustment, there was no significant difference between the two groups in terms of the risk of HCC (Figure 3A; $p=0.478$ ) and CCE (Figure 3B; $p=0.137$ ) development. To adjust bias between the two groups, PS was calculated using the above-mentioned method. PS matching with a 1:1 ratio generated 117 pairs, where variables were well-balanced (all $p>0.05$ ). Similar results were also reproduced; there was no significant difference in terms of the risk of HCC (Figure $4 \mathrm{~A} ; p=0.855$ ) and CCE (Figure 4B; $p=0.437)$ development. 
A

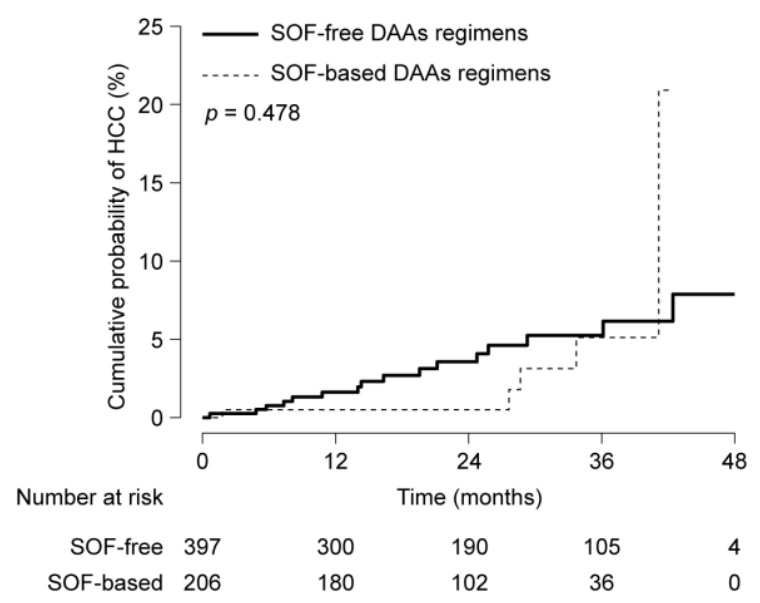

B

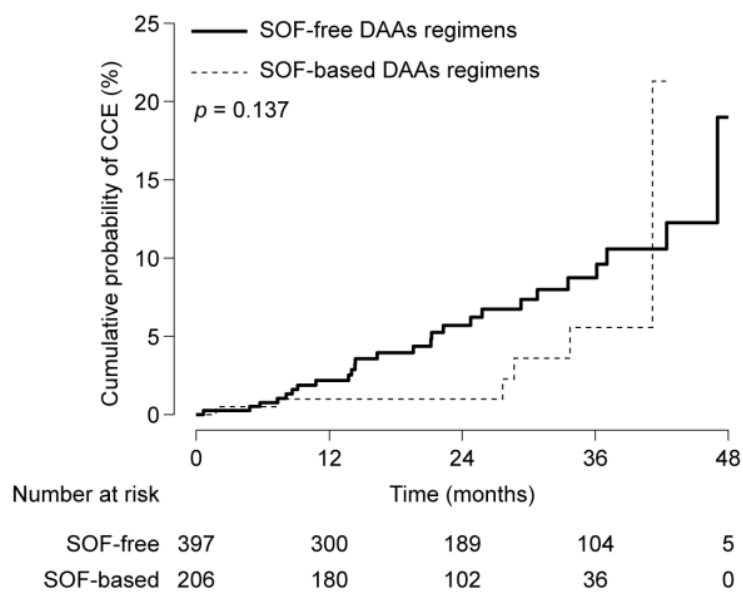

Figure 3. Cumulative probabilities of HCC (A) and CCE (B) development between patients treated with the SOF-based and the SOF-free DAAs regimens before statistical adjustment.

A

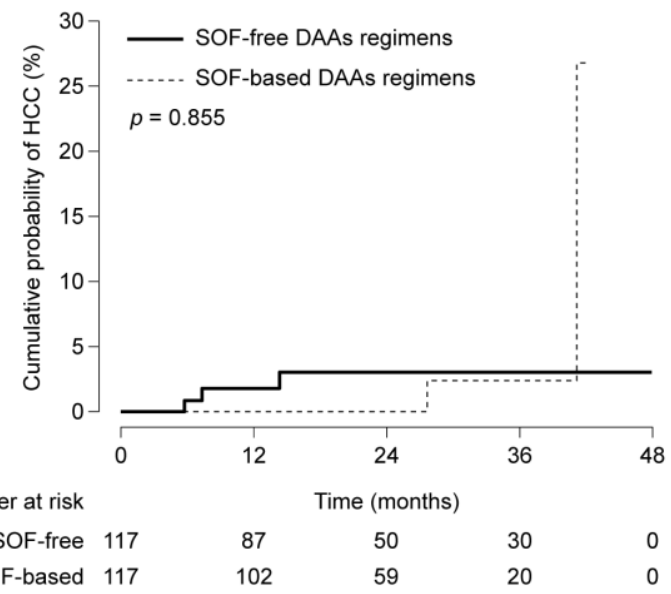

B

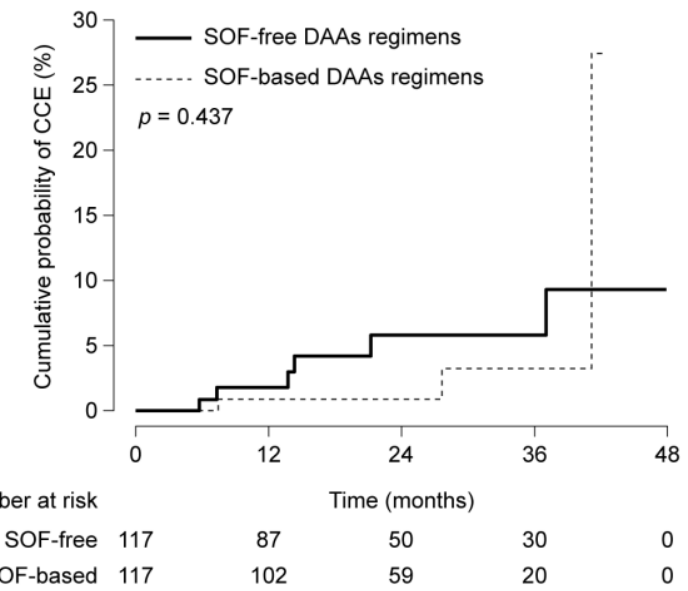

Figure 4. Cumulative probabilities of HCC (A) and CCE (B) development between patients treated with SOF-based and SOF-free DAAs regimens after PS matching analysis. 
Table 4. Comparison of clinical characteristics between patients treated with SOF-based and SOF-free DAA regimens.

\begin{tabular}{|c|c|c|c|}
\hline Variables & SOF-Based DAA Regimens $(n=206)$ & SOF-Free DAA Regimens $(n=397)$ & $p$ Value \\
\hline Age, years & $61.3 \pm 12.2$ & $60.9 \pm 13.1$ & 0.675 \\
\hline Male gender, no. (\%) & $76(36.9)$ & $153(38.5)$ & 0.380 \\
\hline Body mass index, $\mathrm{kg} / \mathrm{m}^{2}$ & $23.7 \pm 3.2$ & $23.8 \pm 3.4$ & 0.747 \\
\hline Diabetes, no. (\%) & $46(22.3)$ & $99(24.9)$ & 0.272 \\
\hline AST, IU/mL & $24.5 \pm 14.7$ & $25.3 \pm 12.9$ & 0.502 \\
\hline ALT, IU/mL & $17.9 \pm 12.3$ & $20.6 \pm 16.1$ & 0.034 \\
\hline Total bilirubin, mg/dL & $1.0 \pm 0.5$ & $0.8 \pm 0.4$ & $<0.001$ \\
\hline Albumin, mg/dL & $4.1 \pm 0.4$ & $4.2 \pm 0.4$ & 0.108 \\
\hline Platelet count, $\times 10^{3} / \mathrm{uL}$ & $201 \pm 76$ & $178 \pm 68$ & $<0.001$ \\
\hline Creatinine, $\mathrm{mg} / \mathrm{dL}$ & $0.8 \pm 0.4$ & $1.0 \pm 1.2$ & $<0.001$ \\
\hline $\mathrm{AFP}, \mathrm{ng} / \mathrm{mL}$ & $3.28(2.38-5.16)$ & $3.24(2.34-4.83)$ & 0.880 \\
\hline FIB-4 index & $1.59(1.15-2.77)$ & $1.88(1.21-2.88)$ & 0.142 \\
\hline Liver cirrhosis, $\mathrm{n}(\%)$ & $55(26.7)$ & $110(27.7)$ & 0.435 \\
\hline
\end{tabular}

Abbreviations: SOF, sofosbuvir; DAAs, direct-acting antivirals; AST, aspartate aminotransferase; ALT, alanine aminotransferase; INR, international normalized ratio. 


\subsection{Risk Stratification According to Post-SVR FIB-4 Index}

In order to identify a subgroup where the universal biannual HCC surveillance program might be safely withdrawn or its interval might be prolonged, we stratified the cumulative risk of HCC development according to a post-SVR FIB-4 index on the basis of another study [10]. Patients with post-SVR FIB-4 index $<2.75(\mathrm{n}=773,71.4 \%)$ and $<3.75(\mathrm{n}=890,82.3 \%$ ) have annual HCC incidences of $<0.2 \%$ /years and $<0.5 \%$ /years, respectively, whereas those with post-SVR FIB- 4 index $\geq 2.75(\mathrm{n}=309,28.6 \%)$ and $\geq 3.75(\mathrm{n}=192,17.7 \%)$ have annual HCC incidences of 3.5\%/years and $4.0 \% / y e a r s$, respectively (Figures S2 and S3, respectively).

The areas under the receiver operating characteristic curve were 0.837 (95\% CI 0.780-0.895) for HCC development and 0.772 (95\% CI 0.704-0.840) for CCE development.

\section{Discussion}

In this study, there was no significant difference in the risk of liver disease progression regarding HCC and CCE development between two groups following statistical adjustment, even though the DAAs group had higher incidences of both HCC and CCE developments than the PegIFN group before adjustment. Notably, before adjustment, the DAAs group had significantly unfavorable pre-treatment parameters, such as older age and a higher proportion of liver cirrhosis, compared to the PegIFN group. In addition, there was no significant difference in clinical prognosis between those undergoing SOF-based and SOF-free DAAs regimens in the present study, despite the in vitro analyses showing that SOF, in comparison with other DAAs, i.e., simeprevir and daclatasvir, tends to activate EGFR-dependent signaling pathways facilitating hepato-carcinogenesis [14]. As the systemic exposure to SOF during antiviral treatment is limited, usually within 12 weeks, its theoretically unfavorable effect may not directly translate into the worse clinical outcomes. However, Rinaldi et al. [31] recently indicated that patients undergoing SOF-based regimens without ribavirin have a 5.7 times higher risk of HCC than those undergoing RBV-based regimens with or without SOF. Hence, future studies regarding the risk of HCC according to the specific type of DAAs regimens will be needed to address such controversies.

Our study has several strengths. First, all of the patients enrolled in our study, including those without underlying advanced fibrosis or cirrhosis, underwent a routine biannual HCC surveillance based upon abdominal ultrasonography and serum alpha-fetoprotein during antiviral treatment and the follow-up after SVR. Therefore, we could establish the appropriate timing of HCC diagnosis, and the HCC incidence observed in our study became much more reliable and practical through prevention of under-estimation [32]. Second, a large sample size $(>1000)$, an appropriate number of clinical events of 3 4\%, given the follow-up duration with a medium term, and the consistently re-produced results after adjustment can provide statistically robust evidence. In a similar context, detailed laboratory data available from a hospital-oriented cohort and a good adherence shown during the follow-up allowed us to evaluate any differences in the HCC incidence more reliably between groups. Last, in order to identify a subgroup where the universal HCC surveillance program might be safely withdrawn or its interval might be prolonged, for example, from bi-annually to annually, we suggested the criteria of post-SVR FIB- 4 index of $<2.75$ according to an annual HCC incidence rate of $<0.2 \%$. By this criteria, approximately $71.4 \%$ of the study population can avoid unnecessary visit to the clinic. To the best of our knowledge, studies assessing HCC risk on the basis of post-SVR FIB-4 index instead of pre-treatment FIB-4 index are relatively scarce so far. Current practice guidelines usually recommend a post-SVR HCC surveillance of high-risk patients, i.e., patients with underlying advanced fibrosis or cirrhosis [33]. However, whether such kinds of HCC screening are necessary among so called "low-risk" patients still remains controversial. For example, the European Association for the Study of the Liver (EASL) recommends that patients with SVR should be discharged unless they have advanced fibrosis/cirrhosis or other risk factors [34]. However, HCC still may occur in so-called low-risk patients after HCV eradication and its risk might persist for $>10$ years after SVR [35]. So, the Asian Pacific Association for the Study of the Liver (APASL) recommends that patients with SVR should be followed 
up at different intervals based on their underlying risks [36]. Further large-scale studies are required to address this issue in terms of not only the medical but also socio-economical perspectives.

The DAAs group had a higher pre-treatment fibrotic burden, estimated through the FIB-4 index, than the PegIFN group. Considering the various disadvantages of PegIFN based regimens, such phenomena might be generally plausible in the practical milieu. The significantly higher proportion of $\mathrm{HCV}$ genotype 2 among the PegIFN group also supported such a trend given the higher SVR rate with the shorter treatment duration for patients with HCV genotype 2. Nevertheless, it is noteworthy that the post-SVR fibrotic burdens between two groups were comparable. Accordingly, the DAAs group showed better anti-fibrotic efficacy expressed as a higher rate of decrease in FIB-4 index than the PegIFN group.

There are several unresolved issues in the present study. First, since this study was conducted using a single tertiary referral hospital-based cohort, the findings are potentially subject to selection bias. However, a homogeneous study population, a statistically reliable sample size and event number, and consistent results through various kinds of statistical adjustment could help to overcome this drawback. Second, in the Republic of Korea, most patients are infected with HCV genotypes $1 \mathrm{~b}$ or 2 [37]. Thus, these results may not be generalizable for the full spectrum of the population with chronic HCV infection, from genotypes 1 to 6 . Therefore, further validation studies are required. Third, given the late timing of reimbursement of DAA regimens in the Republic of Korea, studies with a long-term follow-up duration of $>5$ years are required in the near future in order to assess the risk of HCC development more accurately. In a similar context, to draw a robust conclusion regarding the preventive effect according to the DAA types, further studies including patients treated with newer DAA regimens, i.e., sofosbuvir/velpatasvir or sofosbuvir and velpatasvir/voxilaprevir, are also necessary [34].

\section{Conclusions}

In conclusion, among patients achieving SVR, the overall clinical prognosis was comparable according to antiviral regimens; PegIFN- vs. DAA-based regimens among the entire population and SOF-based vs. SOF-free regimens among the DAAs group. Further studies with a longer follow-up period should be conducted.

Supplementary Materials: The following are available online at http://www.mdpi.com/2072-6694/12/11/3414/s1, Figure S1: Flow chart of study population, Figure S2: Cumulative probabilities of HCC between patients with FIB- $4 \geq 2.75$ and those with FIB- $4<2.75$, Figure S3: Cumulative probabilities of HCC between patients with FIB-4 $\geq 3.75$ and those with FIB- $4<3.75$.

Author Contributions: Conception and design of the study: H.W.L., B.K.K.; Generation, collection, assembly, analysis and/or interpretation of data: H.W.L., D.H.H., H.J.S., J.S.L., S.U.K., J.Y.P., D.Y.K., S.H.A., B.K.K.; Drafting or revision of the manuscript: H.W.L., D.H.H., B.K.K. All authors have read and agreed to the published version of the manuscript.

Funding: This research received no external funding.

Conflicts of Interest: Nothing to declare for all authors.

\section{References}

1. Huang, C.F.; Yu, M.L. Unmet needs of chronic hepatitis C in the era of direct-acting antiviral therapy. Clin. Mol. Hepatol. 2020, 26, 251-260. [CrossRef]

2. Marascio, N.; Quirino, A.; Barreca, G.S.; Galati, L.; Costa, C.; Pisani, V.; Mazzitelli, M.; Matera, G.; Liberto, M.C.; Foca, A.; et al. Discussion on critical points for a tailored therapy to cure hepatitis $C$ virus infection. Clin. Mol. Hepatol. 2019, 25, 30-36. [CrossRef]

3. Molleken, C.; Ahrens, M.; Schlosser, A.; Dietz, J.; Eisenacher, M.; Meyer, H.E.; Schmiegel, W.; Holmskov, U.; Sarrazin, C.; Sorensen, G.L.; et al. Direct-acting antivirals-based therapy decreases hepatic fibrosis serum biomarker microfibrillar-associated protein 4 in hepatitis C patients. Clin. Mol. Hepatol. 2019, 25, 42-51. [CrossRef] [PubMed] 
4. Huang, C.F.; Yu, M.L. Direct-acting antivirals response in hepatocellular carcinoma: Does the presence of hepatocellular carcinoma matter? Clin. Mol. Hepatol. 2019, 25, 168-171. [CrossRef] [PubMed]

5. Parigi, T.L.; Torres, M.C.P.; Aghemo, A. Upcoming direct acting antivirals for hepatitis C patients with a prior treatment failure. Clin. Mol. Hepatol. 2019, 25, 360-365. [CrossRef] [PubMed]

6. Lee, Y.J.; Heo, J.; Kim, D.Y.; Chung, W.J.; Tak, W.Y.; Kim, Y.J.; Paik, S.W.; Sim, E.; Kulasingam, S.; Talwani, R.; et al. An integrated analysis of elbasvir/grazoprevir in Korean patients with hepatitis $C$ virus genotype $1 \mathrm{~b}$ infection. Clin. Mol. Hepatol. 2019, 25, 400-407. [CrossRef]

7. Kim, Y.M.; Kim, S.B.; Song, I.H.; Lee, S.H.; Kim, H.S.; Lee, T.H.; Kang, Y.W.; Kim, S.H.; Lee, B.S.; Chae, H.B.; et al. Efficacy and safety of sofosbuvir plus ribavirin for Korean patients with hepatitis $\mathrm{C}$ virus genotype 2 infection: A retrospective multi-institutional study. Clin. Mol. Hepatol. 2018, 24, 311-318. [CrossRef]

8. Conti, F.; Buonfiglioli, F.; Scuteri, A.; Crespi, C.; Bolondi, L.; Caraceni, P.; Foschi, F.G.; Lenzi, M.; Mazzella, G.; Verucchi, G.; et al. Early occurrence and recurrence of hepatocellular carcinoma in HCV-related cirrhosis treated with direct-acting antivirals. J. Hepatol. 2016, 65, 727-733. [CrossRef]

9. Reig, M.; Marino, Z.; Perello, C.; Inarrairaegui, M.; Ribeiro, A.; Lens, S.; Diaz, A.; Vilana, R.; Darnell, A.; Varela, M.; et al. Unexpected high rate of early tumor recurrence in patients with HCV-related HCC undergoing interferon-free therapy. J. Hepatol. 2016, 65, 719-726. [CrossRef]

10. Farhang Zangneh, H.; Wong, W.W.L.; Sander, B.; Bell, C.M.; Mumtaz, K.; Kowgier, M.; van der Meer, A.J.; Cleary, S.P.; Janssen, H.L.A.; Chan, K.K.W.; et al. Cost effectiveness of hepatocellular carcinoma surveillance after a sustained virologic response to therapy in patients with hepatitis $\mathrm{C}$ virus infection and advanced fibrosis. Clin. Gastroenterol. Hepatol. Off. Clin. Pract. J. Am. Gastroenterol. Assoc. 2019, 17, 1840-1849.e16. [CrossRef]

11. Debes, J.D.; van Tilborg, M.; Groothuismink, Z.M.A.; Hansen, B.E.; Schulze Zur Wiesch, J.; von Felden, J.; de Knegt, R.J.; Boonstra, A. Levels of cytokines in serum associate with development of hepatocellular carcinoma in patients with HCV infection treated with direct-acting antivirals. Gastroenterology 2018, 154, 515-517.e3. [CrossRef] [PubMed]

12. Faillaci, F.; Marzi, L.; Critelli, R.; Milosa, F.; Schepis, F.; Turola, E.; Andreani, S.; Vandelli, G.; Bernabucci, V.; Lei, B.; et al. Liver angiopoietin-2 is a key predictor of de novo or recurrent hepatocellular cancer after hepatitis C virus direct-acting antivirals. Hepatology 2018, 68, 1010-1024. [CrossRef] [PubMed]

13. Giovannini, C.; Fornari, F.; Indio, V.; Trerè, D.; Renzulli, M.; Vasuri, F.; Cescon, M.; Ravaioli, M.; Perrucci, A.; Astolfi, A.; et al. Direct antiviral treatments for hepatitis $C$ virus have off-target effects of oncologic relevance in hepatocellular carcinoma. Cancers 2020, 12, 2674. [CrossRef] [PubMed]

14. Bojkova, D.; Westhaus, S.; Costa, R.; Timmer, L.; Funkenberg, N.; Korencak, M.; Streeck, H.; Vondran, F.; Broering, R.; Heinrichs, S.; et al. Sofosbuvir activates EGFR-dependent pathways in hepatoma cells with implications for liver-related pathological processes. Cells 2020, 9, 1003. [CrossRef]

15. Nagata, H.; Nakagawa, M.; Asahina, Y.; Sato, A.; Asano, Y.; Tsunoda, T.; Miyoshi, M.; Kaneko, S.; Otani, S.; Kawai-Kitahata, F.; et al. Effect of interferon-based and -free therapy on early occurrence and recurrence of hepatocellular carcinoma in chronic hepatitis C. J. Hepatol. 2017, 67, 933-939. [CrossRef]

16. Nahon, P.; Layese, R.; Bourcier, V.; Cagnot, C.; Marcellin, P.; Guyader, D.; Pol, S.; Larrey, D.; De Ledinghen, V.; Ouzan, D.; et al. Incidence of hepatocellular carcinoma after direct antiviral therapy for HCV in patients with cirrhosis included in surveillance programs. Gastroenterology 2018, 155, 1436-1450. [CrossRef]

17. Waziry, R.; Hajarizadeh, B.; Grebely, J.; Amin, J.; Law, M.; Danta, M.; George, J.; Dore, G.J. Hepatocellular carcinoma risk following direct-acting antiviral HCV therapy: A systematic review, meta-analyses, and meta-regression. J. Hepatol. 2017, 67, 1204-1212. [CrossRef]

18. Rinaldi, L.; Nevola, R.; Franci, G.; Perrella, A.; Corvino, G.; Marrone, A.; Berretta, M.; Morone, M.V.; Galdiero, M.; Giordano, M.; et al. Risk of hepatocellular carcinoma after HCV clearance by direct-acting antivirals treatment predictive factors and role of epigenetics. Cancers 2020, 12, 1351. [CrossRef]

19. Ogawa, E.; Nomura, H.; Nakamuta, M.; Furusyo, N.; Kajiwara, E.; Dohmen, K.; Kawano, A.; Ooho, A.; Azuma, K.; Takahashi, K.; et al. Incidence of hepatocellular carcinoma after treatment with sofosbuvir-based or sofosbuvir-free regimens in patients with chronic hepatitis c. Cancers 2020, 12, 2602. [CrossRef]

20. Yeon, J.E. Recent update of the 2017 Korean Association for the Study of the Liver (KASL) treatment guidelines of chronic hepatitis c: Comparison of guidelines from other continents, 2017 AASLD/IDSA and 2016 EASL. Clin. Mol. Hepatol. 2018, 24, 278-293. [CrossRef] 
21. Korean Association for the Study of the Liver. 2017 KASL clinical practice guidelines management of hepatitis c: Treatment of chronic hepatitis c. Clin. Mol. Hepatol. 2018, 24, 169-229. [CrossRef] [PubMed]

22. Korean Association for the Study of the Liver. KASL clinical practice guidelines for liver cirrhosis: Varices, hepatic encephalopathy, and related complications. Clin. Mol. Hepatol. 2020, 26, 83-127.

23. Hanouneh, I.A.; Alkhouri, N.; Singal, A.G. Hepatocellular carcinoma surveillance in the 21st century: Saving lives or causing harm? Clin. Mol. Hepatol. 2019, 25, 264-269. [CrossRef] [PubMed]

24. Na, S.K.; Song, B.C. Development and surveillance of hepatocellular carcinoma in patients with sustained virologic response after antiviral therapy for chronic hepatitis C. Clin. Mol. Hepatol. 2019, 25, 234-244. [CrossRef] [PubMed]

25. Maruyama, H.; Kato, N. Advances in ultrasound diagnosis in chronic liver diseases. Clin. Mol. Hepatol. 2019, 25, 160-167. [CrossRef] [PubMed]

26. Bruix, J.; Sherman, M. Management of hepatocellular carcinoma: An update. Hepatology 2011, 53, 1020-1022. [CrossRef]

27. Kim, T.H.; Kim, S.Y.; Tang, A.; Lee, J.M. Comparison of international guidelines for noninvasive diagnosis of hepatocellular carcinoma: 2018 update. Clin. Mol. Hepatol. 2019, 25, 245-263. [CrossRef]

28. European Association for the Study of the Liver. EASL clinical practice guidelines: Management of hepatocellular carcinoma. J. Hepatol. 2018, 69, 182-236. [CrossRef]

29. Martinez-Chantar, M.L.; Avila, M.A.; Lu, S.C. Hepatocellular carcinoma: Updates in pathogenesis, detection and treatment. Cancers 2020, 12, 2729. [CrossRef]

30. Sterling, R.K.; Lissen, E.; Clumeck, N.; Sola, R.; Correa, M.C.; Montaner, J.; Sulkowski, M.S.; Torriani, F.J.; Dieterich, D.T.; Thomas, D.L.; et al. Development of a simple noninvasive index to predict significant fibrosis in patients with HIV/HCV coinfection. Hepatology 2006, 43, 1317-1325. [CrossRef]

31. Rinaldi, L.; Perrella, A.; Guarino, M.; De Luca, M.; Piai, G.; Coppola, N.; Pafundi, P.C.; Ciardiello, F.; Fasano, M.; Martinelli, E.; et al. Incidence and risk factors of early HCC occurrence in HCV patients treated with direct acting antivirals: A prospective multicentre study. J. Transl. Med. 2019, 17, 292. [CrossRef] [PubMed]

32. Yang, J.D. Detect or not to detect very early stage hepatocellular carcinoma? The western perspective. Clin. Mol. Hepatol. 2019, 25, 335-343. [CrossRef] [PubMed]

33. Galati, G.; Muley, M.; Viganò, M.; Iavarone, M.; Vitale, A.; Dell’Unto, C.; Lai, Q.; Cabibbo, G.; Sacco, R.; Villa, E.; et al. Occurrence of hepatocellular carcinoma after direct-acting antiviral therapy for hepatitis C virus infection: Literature review and risk analysis. Expert Opin. Drug Saf. 2019, 18, 603-610. [CrossRef] [PubMed]

34. Pawlotsky, J.M.; Negro, F.; Aghemo, A.; Berenguer, M.; Dalgard, O.; Dusheiko, G.; Marra, F.; Puoti, M.; Wedemeyer, H.; European Association for the Study of the Liver. EASL recommendations on treatment of hepatitis C: Final update of the series. J. Hepatol. 2020, 73, 1170-1278. [CrossRef] [PubMed]

35. Ioannou, G.N.; Beste, L.A.; Green, P.K.; Singal, A.G.; Tapper, E.B.; Waljee, A.K.; Sterling, R.K.; Feld, J.J.; Kaplan, D.E.; Taddei, T.H.; et al. Increased risk for hepatocellular carcinoma persists up to 10 years after HCV eradication in patients with baseline cirrhosis or high fib-4 scores. Gastroenterology 2019, 157, 1264-1278. [CrossRef]

36. Kanda, T.; Lau, G.K.K.; Wei, L.; Moriyama, M.; Yu, M.L.; Chuang, W.L.; Ibrahim, A.; Lesmana, C.R.A.; Sollano, J.; Kumar, M.; et al. APASL HCV guidelines of virus-eradicated patients by DAA on how to monitor HCC occurrence and HBV reactivation. Hepatol. Int. 2019, 13, 649-661. [CrossRef]

37. Kim, B.K.; Jang, E.S.; Kim, J.H.; Park, S.Y.; Ahn, S.V.; Kim, H.J.; Kim, D.Y. Current status of and strategies for hepatitis C control in South Korea. Clin. Mol. Hepatol. 2017, 23, 212-218. [CrossRef]

Publisher's Note: MDPI stays neutral with regard to jurisdictional claims in published maps and institutional affiliations. 